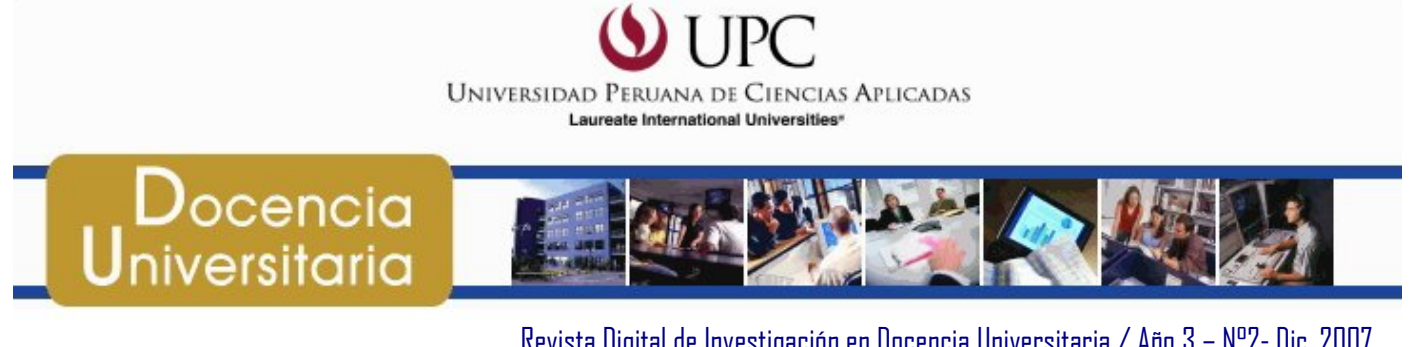

Revista Digital de Investigación en Dacencia Universitaria / Aña 3 - ND2- Dic. 2007

\title{
Tendencias y desafíos para las bibliotecas universitarias peruanas en la gestión del conocimiento académico
}

\author{
Trends and challenges for the Peruvian universities' libraries in \\ the Management of academic knowledge
}

\section{Milagros Angélica Morgan Rozas}

\section{Resumen}

Se identifican tendencias en la gestión del conocimiento académico a las que las bibliotecas universitarias peruanas se vienen dirigiendo. A partir de ellas, se hace una reflexión sobre la esencia de cada una, planteando retos que permitan a las bibliotecas evidenciar su contribución a la mejora de los procesos de enseñanza-aprendizaje, así como a los de generación y difusión del conocimiento científico. Se plantea la hipótesis de que las bibliotecas universitarias peruanas están caminando hacia el modelo del Centro de Recursos para el Aprendizaje y la Investigación (CRAI), sin dejar de ofrecer los servicios que tradicionalmente le son asignados. Para investigar este tema, se utiliza la revisión de las páginas en Internet y de la información publicada en QUELCAS, portal que aglutina una muestra de 32 bibliotecas académicas peruanas; el $56 \%$ de ellas es miembro de ALTAMIRA (Grupo de Bibliotecas Académicas Peruanas), el 36\% se ubica fuera de Lima y el 19\% corresponde a las universidades estatales. Los resultados evidencian diversas tendencias en el rol de las bibliotecas para la gestión del conocimiento académico, demostrando el impacto de las acciones realizadas por ALTAMIRA y señalando varias líneas de trabajo e investigación. Estas contribuirán a desarrollar bibliotecas que respondan de forma proactiva a los nuevos modelos de enseñanza-aprendizaje y de trabajo en red.

Palabras clave: Bibliotecas Universitarias, Gestión del Conocimiento

\section{Abstract}

In the administration of academic knowledge, the trends towards which the Peruvian universities' libraries are steering their ship to are identified. Starting from these, a reflection is made about the reason of being of each one of them and challenges are put forth that allow the libraries to evidence their contribution to the betterment of the teaching and learning processes, as well as the generation and diffusion of scientific knowledge. It begins from the hypothesis that establishes that Peruvian universities' libraries are walking towards a Center of Resources for Learning and Investigation (CRLI) model, without stopping to offer the traditional services that they are normally assigned. To investigate this issue, the revision of Internet web pages is used as a resource, as well as the information published at QUELCAS, the web page for the Peruvian Libraries which corresponds to a sample of 32 academic Peruvian libraries (56\% of which are members of ALTAMIRA, 36\% are located outside the city of Lima and $19 \%$ are state universities). The results evidence diverse trends in the roles of the libraries in the administration of academic knowledge, demonstrate the impact of the actions taken by ALTAMIRA and point to the various lines of work and investigation that will contribute to develop libraries that will respond in a proactive manner to the new models of teaching and learning, as well as networking.

Key words: University libraries, knowledge Management, Peru 


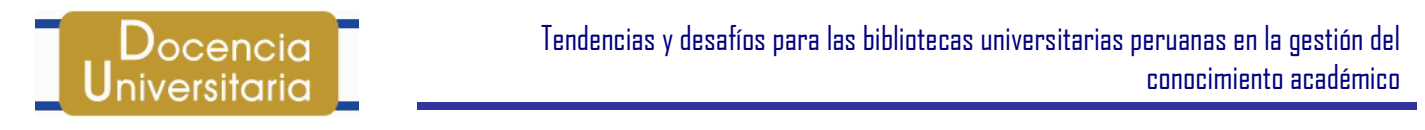

Аก̃̃ 3- N²- Dic. 2007

Para fines del presente trabajo, el término "gestión del conocimiento", o su denominación en inglés, Knowledge Management (KM), se define como el conjunto de procesos que crean, organizan, comparten y aplican el conocimiento para optimizar el logro de la misión y los objetivos de la universidad. De igual forma, se le concibe como el arte de crear valor a partir de una selección de activos del conocimiento (GENG, TOWNLEY, HUANG Y ZHANG 2005: p. 1032)

Tal como señalan Geng, Townley, Huang y Zhang en un estudio comparativo entre universidades norteamericanas y chinas, la disciplina de la gestión del conocimiento (KM) surgió en los años ochenta al reconocerse al conocimiento como una fuerza impulsora de la economía global. Así, los administradores y académicos potenciaron el uso del conocimiento con el fin de incrementar el desempeño de las organizaciones (GENG, TOWNLEY, HUANG Y ZHANG 2005: p. 1032)

Aunque el interés por la gestión del conocimiento se inició en el mundo empresarial, Cronin destaca que sus principios y prácticas pueden ser aplicadas en el sector de las organizaciones de educación superior con similar éxito. Su afirmación se basa en que las universidades son, por excelencia, organizaciones reflexivas e intensivas de conocimiento (CRONIN 2001: p. 130)

Geng, Townley, Huang y Zhang hacen mención a los cinco tipos de conocimiento más utilizados en las organizaciones ${ }^{1}$. El primero es aquel que se encuentra imbuido en las mentes de las personas; es decir, el obtenido a través de la experiencia en el trabajo, incluyendo la investigación, la enseñanza y las actividades operativas. Un segundo tipo de conocimiento es el que se establece en equipos; por ejemplo, las reglas de trabajo del grupo. El tercero es aquel que se documenta y organiza para ser utilizado, tal como el sílabo de un curso. El cuarto tipo de conocimiento es parte de los procesos organizacionales, como la captación de estudiantes o la elaboración de ejercicios de laboratorio. Finalmente, el quinto tipo de conocimiento es aquel que está incluido en los productos y servicios, como las habilidades de los estudiantes graduados, las patentes o las publicaciones. Cada tipo de conocimiento puede ser utilizado para conseguir las misiones y objetivos de las organizaciones (Geng, Townley, Huang y Zhang 2005: p. 1032)

1 Tipología desarrollada por Cross, R., y \& Baird, L. en el año 2000. En: Cross y Baird. Technology is not enough: Improving performance by building organizational memory. Sloan Management Review, 41(3) 2000, 69-78. 


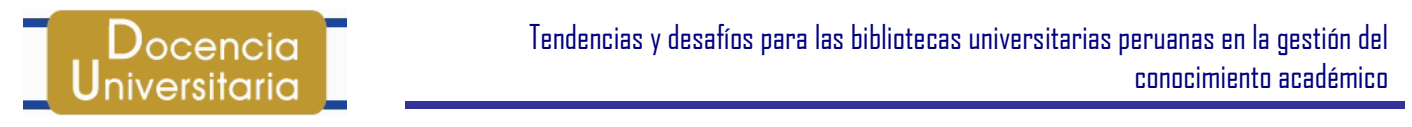

Аก̃̃ 3- N²- Dic. 2007

Para precisar aún más la definición de conocimiento en las universidades, los mismos autores sostienen que este se puede apreciar en dos dimensiones. En primer lugar, en el conocimiento académico que se explicita al ser diseminado por el proceso de enseñanza, la documentación investigativa, las publicaciones, las conferencias, las patentes y las actividades de servicios. La transferencia del conocimiento ocurre en la organización cuando los estudiantes participan en el proceso de enseñanza, cuando los académicos estudian investigaciones publicadas y cuando las distintas disciplinas interactúan, produciéndose una colaboración entre universidades y empresas (Geng, Townley, Huang y Zhang 2005: p. 1032)

De otro lado, el conocimiento operacional es aquel que se genera a partir de las funciones de apoyo, como los servicios de cómputo, los procesos de captación y admisión de alumnos, la contribución a la investigación, los servicios para estudiantes, etc. (Geng, Townley, Huang y Zhang 2005: p. 1033)

De acuerdo a Benavides Velasco, el proceso de gestión del conocimiento incluye las siguientes etapas: identificación y medición del conocimiento; generación del mismo; captura y almacenaje; acceso y transferencia; y, finalmente, aplicación e integración (Benavides 2006: p. 193)

Para todas estas etapas existen disponibles diferentes herramientas que permiten gestionar el conocimiento en una universidad. Geng, Townley, Huang y Zhang hacen referencia a una taxonomía ${ }^{2}$, que incluye manejadores de inteligencia en negocios, instrumentos de trabajo colaborativo, gestores de contenidos y documentos, portales, paquetes de gestión de la relación con el cliente y de minería de datos, manejadores de flujos de trabajo, herramientas de e-learning, sistemas de bibliotecas, sistemas de investigaciones, entre otros (Geng, Townley, Huang y Zhang 2005: p. 1034)

Sobre el rol de las bibliotecas en este proceso de gestión, Huatari y Livonen sostienen que la habilidad competitiva de una universidad mejora cuando su biblioteca es entendida como un aliado estratégico fundamental en la creación de conocimiento. Para que suceda tal situación, se requiere que las bibliotecas asuman un rol más proactivo al interior de la institución, colaborando intensamente con investigadores y profesores, así como con grupos de interés externos (Huatari 2005: p. 328)

Los autores proponen diferentes roles para las bibliotecas universitarias en función de cada una de las categorías en las que se subdividen los procesos del conocimiento: generativo, productivo y representativo. La primera categoría es la que crea nuevo

2 Clasificación planteada por Luan, J. y Serban, A.M. En: Luan y Serban. Technologies, products and models supporting knowledge management. New Directions for Institutional Research, (113) 2002, 85-104. 


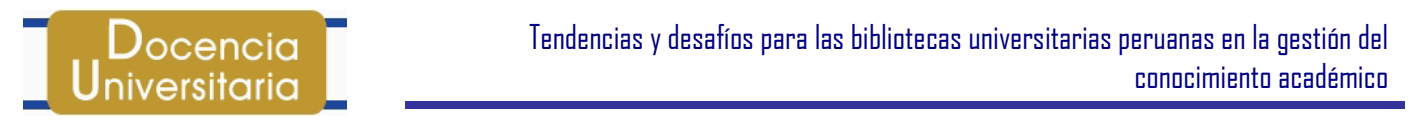

Аก̃̃ 3- N²- Dic. 2007

conocimiento y contribuye a la producción de innovaciones. Las oportunidades de participación de las bibliotecas podrían estar en los servicios de referencia, el desarrollo de colecciones (incluyendo el modelo híbrido de bibliotecas ${ }^{3}$ ), la integración de los programas de desarrollo de competencias informativas con los procesos educativos, la producción de materiales de enseñanza digital, o los espacios de lectura, reflexión y trabajo en grupo (Huatari 2005: p. 325-328)

La segunda categoría corresponde a los procesos del conocimiento productivo, en los que el nuevo conocimiento, proveniente de los procesos generativos, es utilizado con la finalidad de desarrollar o mejorar nuevos productos y servicios. Una oportunidad para las bibliotecas reside, por ejemplo, en el apoyo a los académicos en la gestión de derechos de autor (que les permitan difundir sus investigaciones), en el soporte a los programas de educación a distancia (con servicios de consulta vía correo electrónico), en el desarrollo de tutoriales, entre otros (Huatari 2005: p. 328-329)

La tercera categoría está relacionada al nuevo conocimiento transferido al cliente ${ }^{4}$ con el fin de que este pueda emplearlo como un servicio o producto final. Las oportunidades de participación de las bibliotecas están asociadas a las siguientes actividades: descripción de contenidos; producción de trabajos digitales (como las tesis electrónicas); apoyo a los investigadores y a la universidad en la protección de los derechos de autor de los artículos publicados en revistas pertenecientes a editores privados (tomando en cuenta que luego son cobrados a la universidad cuando dichos editores venden la suscripción a las bibliotecas); y promoción de los beneficios de la publicación de acceso abierto, etc. (HuatarI 2005: p. 329-331)

Desde otra perspectiva, Brenda Cabral visualiza el rol de las bibliotecas como gestoras del conocimiento:

\begin{abstract}
"Uno de los objetivos de la gestión del conocimiento en las bibliotecas es crear conciencia entre el personal de los centros de información acerca de que su labor principal no es solo la de proporcionar información, sino además propiciar que sus usuarios generen conocimientos nuevos. Es decir, hacerlos concientes y hábiles para la innovación, motivarlos para que se mantengan actualizados, haciendo que el conocimiento creado por la institución y por otras sea mejor aprovechado y aplicado a las actividades de la misma. Tendremos así que dirigir nuestras bibliotecas hacia una organización de aprendizaje, y hacer de estas entes que organicen, difundan, transmitan y generen conocimiento. Para ello, la biblioteca tendrá que crear espacios donde se pueda llevar a cabo todo lo anterior”. (Cabral 2006: 16-17)
\end{abstract}

\footnotetext{
3 Se trata de bibliotecas que combinan tanto colecciones y servicios físicos o analógicos como virtuales o digitales.

${ }^{4}$ El cliente es entendido, en su forma más amplia, como beneficiario de los servicios y productos que ofrece la universidad.
} 


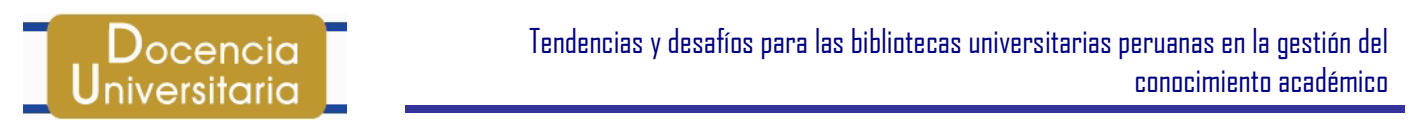

Аก̃̃ 3- N²- Dic. 2007

En cuanto al plano experimental, la bibliografía consultada da cuenta de diversos ejemplos de centros de información o bibliotecas que vienen siguiendo lo expuesto tanto por Huatari y Livonen, como por Brenda Cabral. Esta última cita al Centro Interactivo de Recursos de Información y Aprendizaje (CIRIA) de la Universidad de las Américas (UDLA) en Puebla (México), cuyos servicios se concentran en tres áreas: a) tecnologías avanzadas y personal altamente capacitado para apoyar en las actividades de aprendizaje individual y colaborativo; b) bibliotecas físicas y digitales actualizadas que respaldan los programas académicos y proyectos de investigación; c) programas académicos formales e informales orientados a la administración de información y a la ingeniería del conocimiento (Cabral 2006: p. 17)

Otro modelo hispanoamericano es el de los Centros de Recursos para el Aprendizaje y la Investigación (CRAI), propuestos por la Red de Bibliotecas Universitarias de España (REBIUN):
"Un CRAI es un entorno dinámico, en el que se integran todos los servicios universitarios que dan soporte al aprendizaje y la investigación, relacionados con el mundo de la información y las nuevas tecnologías. (...) Una apuesta innovadora de las bibliotecas para la futura renovación de los métodos de estudio, de docencia y de investigación”. ${ }^{2}$

Asimismo, existen otras experiencias en América Latina respecto a servicios de orientación brindados al alumno, como tutorías en matemáticas, lenguaje y otras materias que requieren mucho repaso. Los CRAI de la Universidad Interamericana de Costa Rica6 constituyen un ejemplo. En el caso peruano, existe un texto que pretende demostrar que el modelo del Centro de Información de la Universidad Peruana de Ciencias Aplicadas (UPC)7 se asemeja a la definición de CRAI planteada por la REBIUN. Cabe mencionar que todos estos conceptos tienen como origen a los Centros de Recursos para el Aprendizaje (Learning Resource Centres), surgidos inicialmente en los colegios y en la enseñanza de idiomas en el Reino Unido.

Analizando las motivaciones que han llevado a construir modelos diferentes, que extienden o complementan el valor insustituible de las bibliotecas, cabe señalar que estas representan una respuesta (o una propuesta de adaptación) a los cambios en la

\footnotetext{
5 Definición encontrada en el Tutor presentado en las II Jornadas REBIUN (Red de Bibliotecas Universitarias Españolas) celebradas en Bilbao en mayo de 2004 Se encuentra en: http://bibliotecnica.upc.es/rebiun/nova/jornadas/segundas_jornadas_rebiun/index.html 6 Ver página en Internet: (www.uicr.edu.cr).

7 Mayor información puede encontrarse en el documento "El Centro de Información de la UPC como potenciador del proceso de enseñanza y aprendizaje en entornos físicos y virtuales”, publicado en “Memorias del XII Coloquio Internacional de Bibliotecarios” (Guadalajara, noviembre de 2005), y elaborado por Liliana Elespuru y Milagros Morgan.
} 


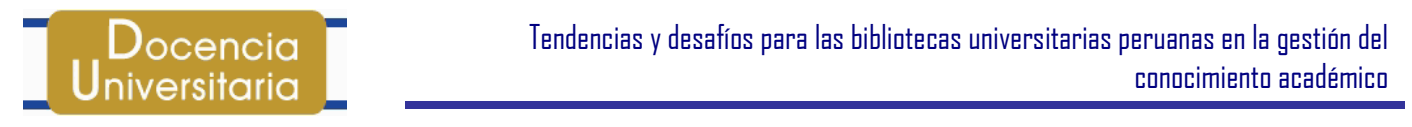

Аก̃̃ 3- N²- Dic. 2007

concepción y métodos de los modelos educativos. Estos han sido fuertemente impulsados por los avances tecnológicos y los descubrimientos en los campos de la psicología, medicina humana y ciencias afines.

Uno de los cambios que más repercusión ha tenido en los modelos educativos es el rol protagónico asumido por el estudiante en su propio aprendizaje. En esta concepción, el alumno se convierte en el centro del proceso educativo, transformándose el profesor en su facilitador u orientador. Por lo tanto, el docente deja de ser el único transmisor de conocimiento en clase, y, por el contrario, es quien abre un mundo de posibilidades al alumno, trascendiendo, incluso, al espacio del aula. Un ejemplo es la metodología del Aprendizaje Basado en Problemas (ABP), en la que el profesor no dicta clases, encargándose solo de definir los temas de investigación; los estudiantes, por su parte, recurren a las bibliotecas para encontrar las soluciones a dichos problemas, desarrollando así el dominio de una materia o campo de estudio específico. Los alumnos se reúnen luego con el docente para revisar las fuentes consultadas, evaluando su nivel de aprendizaje respecto a las soluciones encontradas o cuestiones planteadas.

A pesar de todos los cambios descritos, existen ciertas condiciones inherentes a toda universidad que prevalecen a lo largo del tiempo. Luis Jaime Cisneros, destacado profesor universitario peruano, las ha descrito en una columna del diario La República, señalando lo siguiente:

“(...) ahora llegan a la institución superior alumnos desacostumbrados a la lectura y a la reflexión. (...) Pero la universidad necesita que los estudiantes sepan leer con claridad (...). El libro debe hacer comprender al alumno que la crítica, la reflexión y el debate son instrumentos indispensables para organizar el saber y perfeccionar la técnica: gracias a eso el libro estimula la capacidad creadora y enriquece espiritualmente al individuo". 8

El presente estudio tiene como objetivo identificar posibles tendencias en los roles de las bibliotecas académicas peruanas frente a la gestión del conocimiento académico y, a su vez, establecer desafíos que provoquen una mejora sustancial en su contribución a dicho proceso.

Con fines metodológicos, se utilizará la siguiente hipótesis de trabajo: las bibliotecas académicas peruanas están caminando hacia el modelo del Centro de Recursos para el Aprendizaje y la Investigación (CRAI), sin dejar de ofrecer los servicios que tradicionalmente le son asignados.

8 Título del artículo “la universidad y la lectura”. Diario La República, 19 de agosto de 2007, página 23. 


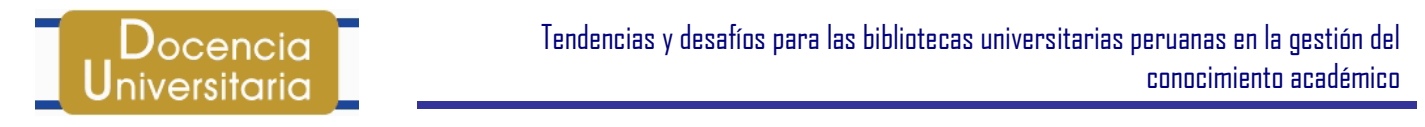

Аก̃̃ 3- N²- Dic. 2007

Para comprobar o refutar tal hipótesis, se realizó una revisión de páginas de Internet y del directorio de bibliotecas de QUELCAS, Portal de las Bibliotecas Peruanas ${ }^{9}$, que aglutina a 32 bibliotecas académicas del país. Se eligió dicha metodología por tres razones: a) se trata de información que es pública, considerándola como declaración oficial de los servicios que ofrecen; b) disponibilidad de tiempo para realizar la investigación; c) preocupación por la baja tasa de respuesta que reciben los cuestionarios enviados por correo.

Los criterios empleados para elegir la muestra de 32 bibliotecas fueron los siguientes: se consideró a todos los miembros de ALTAMIRA, Grupo de Bibliotecas Académicas Peruanas $^{10}$ y a todas las bibliotecas de educación superior que registraron su información en QUELCAS (se observó que el directorio no estaba actualizado y por tanto, se decidió revisar las páginas en Internet de cada una de las bibliotecas seleccionadas) A continuación, se precisa la conformación de la muestra ${ }^{11}$ :

Cuadro 1: Conformación de la muestra

\begin{tabular}{|c|c|c|c|c|c|c|c|}
\hline $\begin{array}{c}\text { Con } \\
\text { información } \\
\text { en } \\
\text { QUELCAS }\end{array}$ & $\begin{array}{l}\text { Miembro de } \\
\text { ALTAMIRA }\end{array}$ & $\begin{array}{l}\text { Fuera de } \\
\text { Lima }\end{array}$ & $\begin{array}{c}\text { Universidad } \\
\text { Privada }\end{array}$ & $\begin{array}{c}\text { Universidad } \\
\text { Nacional }\end{array}$ & $\begin{array}{c}\text { Total } \\
\text { universidades }\end{array}$ & $\begin{array}{c}\text { Otra } \\
\text { institución } \\
\text { académica }\end{array}$ & $\begin{array}{c}\text { Total } \\
\text { muestra }\end{array}$ \\
\hline 24 & 18 & 8 & 16 & 6 & 22 & 10 & 32 \\
\hline $75 \%$ & $56 \%$ & $36 \%$ & $73 \%$ & $19 \%$ & $69 \%$ & $31 \%$ & $100 \%$ \\
\hline
\end{tabular}

Cabe señalar que la selección de los miembros de ALTAMIRA obedeció a que esta institución agrupa a todos los tipos de universidades peruanas (públicas, privadas, ubicadas dentro y fuera de Lima, etc.) y porque se esperó hallar evidencias de su impacto en la mejora de las colecciones y servicios que ofrecen las bibliotecas peruanas.

Respecto al total de las universidades peruanas, la muestra representó al 25\%, de acuerdo a lo precisado en la página Web de la Asamblea Nacional de Rectores (ANR) ${ }^{12}$.

\footnotetext{
9 (http://quelcas.rcp.net.pe/bibliotecas.php.). En el Directorio de Bibliotecas de QUELCAS, estas ingresan su información de forma espontánea, actualizándola de manera autónoma dado que reciben una contraseña individual para acceder en el momento que requieran modificar o agregar algún dato. 10 (http://www.universia.edu.pe/altamira/). ALTAMIRA se creó formalmente en el último trimestre de 2001. Sin embargo, sus actividades se remontan a la firma, en 1999, de un acuerdo de cooperación para la adquisición de bases de datos de revistas digitales.

11 Para fines de extender o profundizar la presente investigación, sí será posible mostrar al interesado la plantilla de instituciones analizadas.

12 (http://www.anr.edu.pe).
} 
Cuadro 2: Universo del Estudio

\begin{tabular}{|c|c|c|c|c|c|c|}
\hline $\begin{array}{c}\text { Total } \\
\text { universo }\end{array}$ & $\begin{array}{c}\text { Universidad } \\
\text { pública bajo } \\
\text { jurisdicción } \\
\text { de la ANR }\end{array}$ & $\begin{array}{c}\text { Universidad } \\
\text { privada bajo } \\
\text { jurisdicción } \\
\text { de la ANR }\end{array}$ & $\begin{array}{c}\text { Universidad } \\
\text { privada bajo } \\
\text { jurisdicción } \\
\text { de la } \\
\text { CONAFU }\end{array}$ & $\begin{array}{c}\text { Muestra total } \\
\text { de } \\
\text { universidades }\end{array}$ & $\begin{array}{c}\text { Muestra de } \\
\text { universidades } \\
\text { públicas }\end{array}$ & $\begin{array}{c}\text { Muestra de } \\
\text { universidades } \\
\text { privadas }\end{array}$ \\
\hline 89 & 28 & 40 & 21 & 22 & 6 & 16 \\
\hline $100 \%$ & $31 \%$ & $45 \%$ & $24 \%$ & $25 \%$ & $21 \%$ & $26 \%$ \\
\hline
\end{tabular}

Si se extrapola a la muestra el porcentaje de universidades públicas y privadas correspondientes al universo, se puede apreciar que las proporciones fueron bastante cercanas a la realidad, tal como se observa en el siguiente cuadro:

Cuadro 3: Comparación entre el universo y la muestra del Estudio

\begin{tabular}{|c|c|c|}
\hline Tipo & $\#$ & $\%$ \\
\hline Universo & 89 & $100 \%$ \\
\hline Públicas & 28 & $31 \%$ \\
\hline Privadas & 61 & $69 \%$ \\
\hline Muestra & 22 & $100 \%$ \\
\hline Públicas & 6.82 & $31 \%$ \\
\hline Privadas & 15.18 & $69 \%$ \\
\hline
\end{tabular}

Es cierto que existió una limitación en el presente estudio, ya que solo abarcó a las bibliotecas universitarias que contaban con una página Web o que habían publicado su información en QUELCAS. Esto obedeció únicamente a razones de tiempo, por lo que se sugiere continuar esta investigación, complementándola con llamadas telefónicas y visitas de campo.

Respecto a las instituciones académicas no adscritas a una universidad, se encontró oportuno tomar en cuenta a diez de ellas ${ }^{13}$ por las siguientes razones: a) su cercanía con

\footnotetext{
${ }^{13}$ El 100\% había colocado su información en QUELCAS.
} 


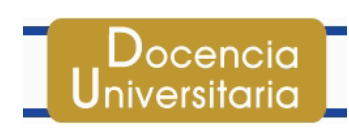

los programas de investigación realizados por las universidades; b) su atención a los estudiantes, al contar con colecciones especializadas que no necesariamente pueden encontrarse en las bibliotecas universitarias; y c) que pertenecieran a ALTAMIRA.

Para la recolección de datos, se elaboró una matriz en Excel que permitió registrar la data de cada biblioteca y compararla con el resto de integrantes de la muestra.

Los datos recolectados correspondieron a elementos que daban indicios sobre el rol que las bibliotecas académicas cumplían en sus respectivas instituciones. Dichos factores fueron agrupados en las siguientes categorías: tipo de servicios; tipo de colecciones; tipo de estantería; ambientes o instalaciones físicas; y utilización de sistemas de gestión del conocimiento académico.

Los resultados por tipo de servicios arrojaron un predominio de los servicios tradicionales de una biblioteca académica, como la lectura en sala (97\%), referencia (81\%), y el acceso virtual al catálogo en línea (78\%). Este último dato resultó interesante, tomando en cuenta que han pasado solo doce años desde que el primer catálogo de una biblioteca universitaria peruana fuera colocado en Internet.

Cuadro 4: Tipo de Servicios

\begin{tabular}{|c|c|c|c|c|c|c|c|c|c|c|c|}
\hline \multicolumn{12}{|c|}{ Tipo de servicios } \\
\hline 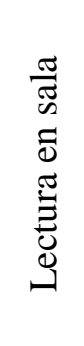 & 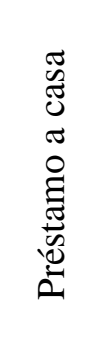 & 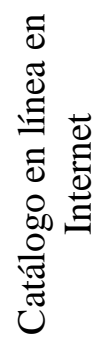 & 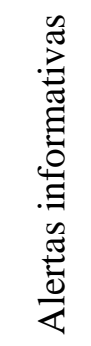 & 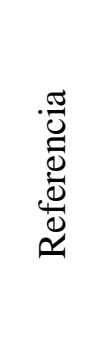 & 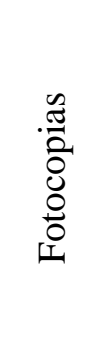 & 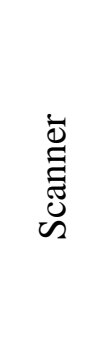 & 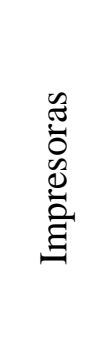 & 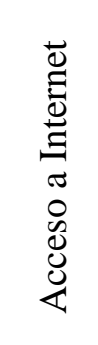 & 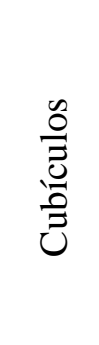 & 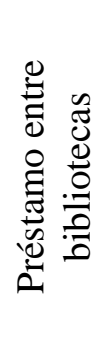 & 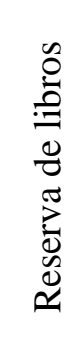 \\
\hline 31 & 21 & 25 & 21 & 26 & 20 & 10 & 11 & 18 & 6 & 18 & 10 \\
\hline $97 \%$ & $66 \%$ & $78 \%$ & $66 \%$ & $81 \%$ & $63 \%$ & $31 \%$ & $34 \%$ & $56 \%$ & $19 \%$ & $56 \%$ & $31 \%$ \\
\hline
\end{tabular}

Otro elemento que llamó la atención fue que un 56\% ofreciera acceso a Internet a sus estudiantes dentro de los ambientes de la biblioteca, así como que un 34\% diera el servicio de impresoras, y un 31\% el de scanner. En relación a los cubículos o salas de 


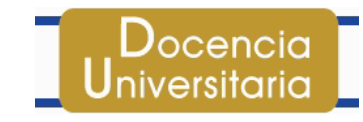

trabajo en grupo, se apreció que solo un 19\% de las bibliotecas ofrecía esta facilidad. Sin embargo, se encontró una biblioteca que contaba con 37 cubículos equipados con computadoras multimedia y conexión a Internet.

Los resultados por tipo de colecciones permitieron observar que también predominaba la colección impresa de libros y revistas (97\%), seguida de un 75\% que contaba con discos compactos, un $69 \%$ que incluía videos (tanto en formato VHS como DVD) ${ }^{14}$, y un $66 \%$ que ofrecía revistas digitales. La proporción de un 34\% de bibliotecas que contaban con libros digitales es un aspecto que debe monitorearse en próximos estudios.

Cuadro 5: Tipo de documentos

\begin{tabular}{|c|c|c|c|c|c|c|c|c|}
\hline \multicolumn{9}{|c|}{ Tipo de documentos } \\
\hline 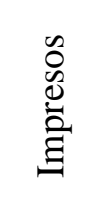 & త & 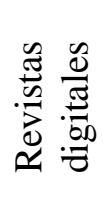 & 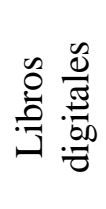 & 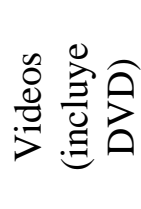 & 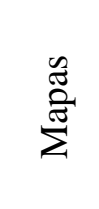 & $\begin{array}{l}\mathscr{O} \\
\frac{\pi}{\tilde{a}}\end{array}$ & 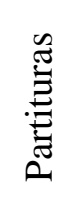 & 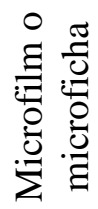 \\
\hline 31 & 24 & 21 & 11 & 22 & 15 & 6 & 1 & 4 \\
\hline $97 \%$ & $75 \%$ & $66 \%$ & $34 \%$ & $69 \%$ & $47 \%$ & $19 \%$ & $3 \%$ & $13 \%$ \\
\hline
\end{tabular}

Con el fin de conocer si se estaba suscitando un cambio en la forma de acceso a las colecciones impresas (para acercar a los usuarios a los libros), se constató que, hasta esa fecha, la mayoría de bibliotecas seguía manteniendo el esquema de estantería cerrada (63\%).

Cuadro 6: Tipo de Estantería

\begin{tabular}{|c|c|c|}
\hline \multicolumn{3}{|c|}{ Estantería } \\
\hline Abierta & Cerrada & Mixta \\
\hline 5 & 20 & 4 \\
\hline $16 \%$ & $63 \%$ & $13 \%$ \\
\hline
\end{tabular}

En lo concerniente a la incorporación de diferentes facilidades físicas o tecnológicas dentro de los ambientes de las bibliotecas, se evidenció que solo algunas habían realizado modificaciones sobre el particular:

14 No se ha podido establecer el porcentaje de la colección de videos correspondiente a DVD o a VHS. 


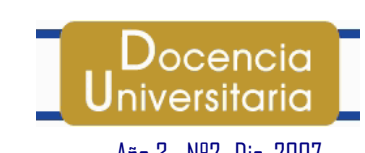

Aก̃̃ 3- N02- Dic. 2007

Cuadro 7: Facilidades físicas y tecnológicas

\begin{tabular}{|c|c|c|c|c|c|}
\hline $\begin{array}{c}\text { Sala de } \\
\text { Capacitación }\end{array}$ & $\begin{array}{c}\text { Sala para } \\
\text { profesores }\end{array}$ & $\begin{array}{c}\text { Sala de } \\
\text { conferencias o } \\
\text { auditorio }\end{array}$ & $\begin{array}{c}\text { Librería } \\
\text { dentro de la } \\
\text { biblioteca }\end{array}$ & $\begin{array}{c}\text { Cafetería } \\
\text { integrada a la } \\
\text { biblioteca }\end{array}$ & WI-FI \\
\hline 3 & 2 & 1 & 3 & 1 & 4 \\
\hline $9 \%$ & $6 \%$ & $3 \%$ & $9 \%$ & $3 \%$ & $13 \%$ \\
\hline
\end{tabular}

En la revisión de la página Web de una de las bibliotecas consideradas en la muestra, se encontró que contaba con una sala de capacitación equipada con 20 computadoras y proyección multimedia. Indicaba que allí se realizaban talleres de desarrollo de habilidades informativas dirigidos a profesores y estudiantes, así como sesiones de capacitación en el manejo de herramientas tecnológicas aplicables al proceso de enseñanza-aprendizaje. Asimismo, contaba con una sala de uso exclusivo para profesores, la cual estaba equipada con 27 computadoras y dos cubículos para el trabajo en grupo.

El cuarto elemento investigado fue el manejo de sistemas de gestión del conocimiento académico:

Cuadro 8: Sistemas de automatización

\begin{tabular}{|c|c|c|c|c|}
\hline $\begin{array}{c}\text { Tesis FT en la Web } \\
\text { o Intranet }\end{array}$ & $\begin{array}{c}\text { Gestor de } \\
\text { contenidos de la } \\
\text { Universidad }\end{array}$ & $\begin{array}{c}\text { Sistema } \\
\text { Integrado (ILS) }\end{array}$ & $\begin{array}{c}\text { Auto } \\
\text { préstamo }\end{array}$ & $\begin{array}{c}\text { Meta- } \\
\text { buscador }\end{array}$ \\
\hline 5 & 2 & 5 & 1 & 1 \\
\hline $16 \%$ & $6 \%$ & $16 \%$ & $3 \%$ & $3 \%$ \\
\hline
\end{tabular}

Al respecto, pudo apreciarse que la publicación digital de tesis (16\%) y el uso de un sistema integrado para bibliotecas (16\%) eran los aplicativos que tenían mayor incidencia, en comparación con los gestores de contenidos, los metabuscadores y el autopréstamo. 


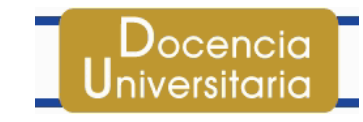

En lo referente a las tesis, pudo averiguarse que tres bibliotecas participaban en el proyecto Cybertesis ${ }^{15}$. En cuanto a las dos bibliotecas que no participaban en Cybertesis, una brindaba acceso a las mismas solo a través de su Intranet; la otra desarrollada una metodología propia en la Web. De las que participaban en Cybertesis, solo una había conseguido que los estudiantes redactaran o elaboraran sus tesis en la plantilla propia del sistema, limitándose la biblioteca a brindar el servicio de capacitación, verificar el proceso de marcación y realizar la conversión para su publicación digital.

Con relación a los sistemas integrados ${ }^{16}$, se observó un incremento en el número de bibliotecas que contaba con uno. Sin embargo, se trataba de un grupo pequeño. Las cinco bibliotecas tenían un sistema integrado que había sido suministrado por un proveedor internacional. Cabe señalar que, a pesar de no contar con este tipo de sistemas, el 78\% de las bibliotecas sí ofrecía el acceso a su catálogo en línea a través de su página Web.

El último factor de análisis fue el de las funciones o responsabilidades adicionales que asumían las bibliotecas. En el siguiente cuadro, se puede apreciar que los programas de formación de usuarios eran los que se desarrollaban en un porcentaje más alto (15\%), pero del mismo modo, se trataba de un grupo muy reducido. Llamó la atención que dos bibliotecas tuvieran a su cargo la gestión de la editorial universitaria, así como que otras dos realizaran la edición de videos para sus usuarios.

Cuadro 9: Funciones adicionales asignadas a las bibliotecas

\begin{tabular}{|c|c|c|c|c|}
\hline $\begin{array}{c}\text { Asesoría a } \\
\text { profesores en } \\
\text { elaboración de } \\
\text { materiales de } \\
\text { clase }\end{array}$ & $\begin{array}{c}\text { Grabación de } \\
\text { clases y } \\
\text { visualización en } \\
\text { biblioteca }\end{array}$ & $\begin{array}{c}\text { Edición de } \\
\text { videos }\end{array}$ & $\begin{array}{c}\text { Programa de } \\
\text { formación de } \\
\text { usuarios }\end{array}$ & $\begin{array}{c}\text { Gestión del } \\
\text { Fondo Editorial }\end{array}$ \\
\hline 1 & 1 & 2 & 5 & 2 \\
\hline $3 \%$ & $3 \%$ & $6 \%$ & $16 \%$ & $6 \%$ \\
\hline
\end{tabular}

Luego de mostrar y analizar los resultados del presente estudio, se pasará a verificar la hipótesis del trabajo. En tal sentido, no es posible afirmar que, en la actualidad, las

\footnotetext{
${ }^{15}$ Portal de las tesis de las universidades: iniciativa promovida por la UNESCO y varias universidades en el mundo. En el Perú, la UNMSM es responsable de su aplicación y desarrollo. URL:

(http://www.cybertesis.net).

16 Solución tecnológica o sistema que integra todos los procesos de una biblioteca (adquisiciones, catalogación, clasificación e indización, búsquedas, préstamos, devoluciones, control de usuarios, etc.).
} 


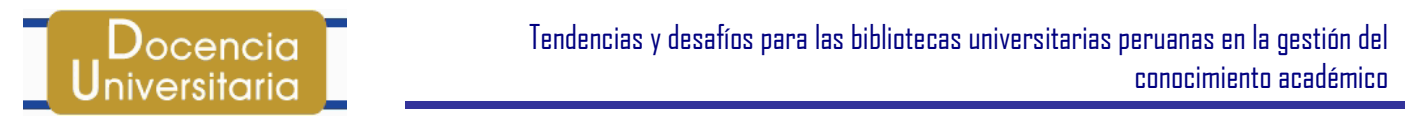

Аก̃̃ 3- N²- Dic. 2007

bibliotecas académicas peruanas estén caminando hacia el modelo del Centro de Recursos para el Aprendizaje y la Investigación (CRAI). Sin embargo, sí se puede concluir que estas siguen ofreciendo los servicios que tradicionalmente les han sido asignados, y que, además, pueden ser catalogadas como bibliotecas híbridas, en tanto incorporan contenidos digitales a sus colecciones.

De otro lado, también es posible sustentar que existen algunos indicios de cambio en las concepciones, funciones, servicios, composición de colecciones, etc., pero que se encuentran en una fase aún incipiente, suscitándose apenas en un grupo pequeño de bibliotecas.

Tales indicios se evidencian en el incremento de colecciones de revistas digitales, así como en el servicio de acceso a Internet dado al interior de los ambientes de la biblioteca. Sin embargo, faltaría constatar si este acceso no solo permite la consulta de colecciones digitales, sino también si se pueden emplear las computadoras para elaborar trabajos e investigaciones.

El hecho que un 66\% de bibliotecas cuenten con revistas digitales, y que un 34\% tenga libros electrónicos, obedece a la existencia de ALTAMIRA y de otros grupos que han propiciado la adquisición cooperativa de este tipo de colecciones. En 1999, año en que los miembros fundadores de ALTAMIRA comenzaron a reunirse, solo existían dos universidades peruanas (una pública y otra privada) que contaban con revistas digitales en formato de disco compacto, y no vía Internet, como sucede en la actualidad.

Aunque son pocas las bibliotecas que los han desarrollado, la adquisición de sistemas integrados da señales de una madurez y un crecimiento de sus operaciones, dado que son sistemas robustos que funcionan y que han sido probados en distintos centros de información en el mundo, presentando altos niveles de transacción (búsquedas, préstamos, devoluciones, catalogación de documentos, etc.).

El presente estudio es un primer intento de conocer las tendencias en el rol de las bibliotecas académicas peruanas frente a la gestión del conocimiento. Por lo tanto, se recomienda continuarlo con investigaciones que comparen su evolución en el tiempo, extendiendo el universo de estudio, y realizando cruces de variables a fin de hallar correlaciones y patrones de comportamiento.

Se sugiere a ALTAMIRA que analice la posibilidad de relanzar QUELCAS, con el objetivo de ofrecer al mundo referencias actualizadas sobre las bibliotecas peruanas, además de incluir en Internet a aquellas bibliotecas académicas que aún no cuentan con una página propia. 


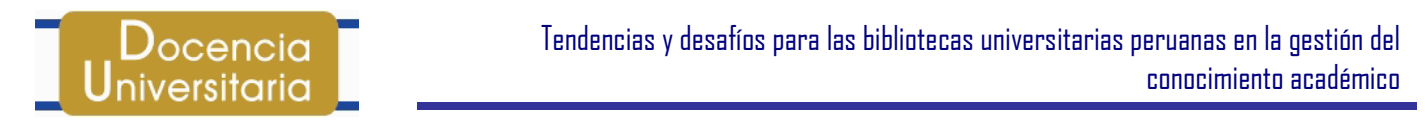

Аก̃o 3- N22- Dіс. 2007

Finalmente, se plantean los siguientes desafíos para las bibliotecas académicas peruanas:

- Las bibliotecas son expertas en la selección, organización, difusión y apoyo a la generación de nuevo conocimiento. Por lo tanto, se sugiere explorar nuevas áreas o aspectos en los que se pueda contribuir a la gestión del conocimiento académico en la universidad, trascendiendo su labor tradicional. Esto implica pensar, por ejemplo, en el rol de las bibliotecas frente a los nuevos modelos de enseñanza semi presencial y totalmente a distancia.

- $\quad$ Se sugiere a cada biblioteca reflexionar sobre su papel respecto a la gestión del conocimiento académico. Se considera que no existe ni debe existir un único modelo de biblioteca académica; cada una debe elegir, concientemente y de forma reflexiva y sistemática, el modelo que más se acomode a los procesos de gestión del conocimiento de sus propias universidades, ya que cada una tiene distintas misiones y visiones. Dicha reflexión implica la identificación de fortalezas y aspectos a mejorar, con el fin de establecer hasta dónde pueden comprometerse con el proceso.

- Las coincidencias que existan permitirán establecer ciertos patrones comunes, debido a que todas las universidades se encuentran comprometidas con el desarrollo de las personas y de la sociedad.

- Se plantea seguir trabajando de forma cooperativa y encontrar nuevas posibilidades para el desarrollo de todas las bibliotecas académicas peruanas, teniendo en consideración un entorno cada vez más competitivo en el sector de la educación superior, no solo dentro, sino fuera del Perú.

- $\quad$ Es imprescindible buscar mecanismos que permitan a las bibliotecas ser más efectivas y eficientes, demostrando el impacto de sus servicios en la mejora de la calidad de la enseñanza, y aprovechando al máximo los recursos disponibles. Es una necesidad, por lo tanto, que las bibliotecas participen activamente en los procesos de autoevaluación, acreditación y certificación de la calidad. 


\section{Bibliografía}

BENAVIDES VELASCO, Carlos A.

2006 Gestión del conocimiento y calidad total. España: Díaz de Santos.

CABRAL VARGAS, Brenda.

2006 El papel de las bibliotecas y la educación en la gestión del conocimiento de la sociedad contemporánea. Alexandria, 3 (6): 15-19.

CRONIN, Blaise.

2001 Knowledge management, organizational culture and Anglo-American higher education. Journal of Information Science, 27(3): 129-137

GENG, Q., TOWNLEY, C., HUANG, K., \& ZHANG, J.

2005 Comparative knowledge management: A pilot study of chinese and american universities. Journal of the American Society for Information Science and Technology, 56(10): 1031-1044.

HUOTARI, M., \& IIVONEN, M.

2005 Knowledge processes: A strategic foundation for the partnership between the university and its library. Library Management, 26(6/7): 324-335.

Para citar este documento, puede utilizar la siguiente referencia:

MORGAN, Milagros (2007). "Tendencias y desafíos para las bibliotecas universitarias peruanas en la gestión del conocimiento académico”[artículo en línea]. Revista Digital de Investigación en Docencia Universitaria (RIDU)

Año 3- $N^{\circ} 2$ - Diciembre2007.. [Fecha de consulta: dd/mm/aa].

$<$ http://beta.upc.edu.pe/calidadeducativa/ridu/2006/ridu4_5MM.pdf >>

\section{Milagros Morgan Rozas}

\section{mmorgan@upc.edu.pe}

Magíster en Administración de Empresas de la Universidad Peruana de Ciencias Aplicadas (UPC) y MBA de la Universidad Politécnica de Cataluña (España). Licenciada en Bibliotecología y Ciencia de la Información de la Pontificia Universidad Católica del Perú. Presidenta del Consejo Directivo de ALTAMIRA, Grupo de Bibliotecas Peruanas. Actualmente es responsable de la Gerencia del Fono Editorial de la UPC y Directora del Centro de Información de la Universidad Peruana de Ciencias Aplicadas (UPC). 